\title{
BMJ Open Cardiovascular and neuropsychiatric safety of varenicline and bupropion compared with nicotine replacement therapy for smoking cessation: study protocol of a retrospective cohort study using the QResearch general practice database
}

\author{
Daniel Kotz, ${ }^{1,2,3}$ Colin Simpson, ${ }^{3}$ Wolfgang Viechtbauer, ${ }^{4}$ \\ Onno C P van Schayck, ${ }^{1,3}$ Robert West, ${ }^{2}$ Aziz Sheikh ${ }^{1,3,5}$
}

To cite: Kotz D, Simpson C, Viechtbauer W, et al. Cardiovascular and neuropsychiatric safety of varenicline and bupropion compared with nicotine replacement therapy for smoking cessation: study protocol of a retrospective cohort study using the QResearch general practice database. BMJ Open 2014;4: e005281. doi:10.1136/ bmjopen-2014-005281

- Prepublication history and additional material is available. To view please visit the journal (http://dx.doi.org/ 10.1136/bmjopen-2014005281).

Received 18 March 2014 Revised 15 July 2014 Accepted 17 July 2014

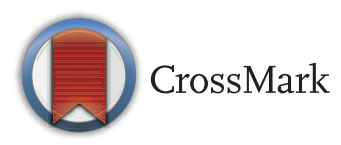

For numbered affiliations see end of article.

Correspondence to

Dr Daniel Kotz;

d.kotz@maastrichtuniversity.

nl, http://www.daniel-kotz.de

\section{ABSTRACT}

Introduction: Cigarette smoking continues to be the leading cause of preventable death and is the main risk factor of major diseases such as chronic obstructive pulmonary disease (COPD). The best treatment to help smokers quit is a combination of behavioural support with pharmacotherapy. Varenicline is the newest drug on the market and has been shown to be effective in the general smoking population and in smokers with COPD. The safety profile of varenicline was initially established using standard approaches to pharmacovigilance, but postmarketing reports have raised concerns about a possible association between the use of varenicline and cardiovascular and neuropsychiatric events. Although recent studies have not confirmed such an association, further research is needed given the large number of smokers who are being prescribed varenicline, including important subgroups such as smokers with COPD who may be particularly vulnerable to side effects of drugs. The aim of this study is to assess the cardiovascular and neuropsychiatric safety of varenicline using data from the QResearch general practice (GP) database.

Methods and analysis: We will conduct a retrospective cohort study in the QResearch GP database. Patients will be categorised into three exposure groups: prescription of (1) varenicline, (2) bupropion or (3) nicotine replacement therapy (NRT Rx; =reference group). We will separately consider major incident neuropsychiatric and cardiovascular outcomes that occur during 6 months of follow-up using Cox proportional hazards models, adjusted for confounders. Furthermore, propensity score analysis will be used as an analytical approach to account for potential confounding by indication.

Ethics and dissemination: This work involves analysis of anonymised, routinely collected data. The protocol has been independently peer-reviewed by

\section{Strengths and limitations of this study}

- The proposed study uses routinely collected data from a general practice database. The large sample size will allow the investigation of rare adverse events that may be associated with medication usage.

- Unrestricted inclusion of all patients from a very large general practice population will mean that findings from this study will be highly generalisability to other populations.

- As we are using an observational study design and treatment allocation is non-randomised, bias in the form of confounding by indication will be present. We will therefore use different statistical techniques such as propensity scores to adjust our findings for the most important confounders.

- A disadvantage of using routinely collected general practices is that some variables of potential interest may be missing and/or of poor quality.

the QResearch Scientific Board and meets the requirements of the Trent research ethics committee. We plan to disseminate the results from this study via articles in international peer-reviewed journals and presentations at relevant national and international health conferences.

\section{INTRODUCTION}

Cigarette smoking continues to be the leading cause of preventable death, killing nearly six million people worldwide each year. ${ }^{1}$ Smokers who do not stop lose at least one decade of life expectancy. ${ }^{2}$ Chronic obstructive pulmonary 
disease (COPD) is one of the major causes of death in smokers and mortality rates are still increasing. ${ }^{2} 3$ The WHO estimates that about 3.3 million people die from COPD worldwide each year and this figure is expected to rise substantially in the coming decades. 4

It has been estimated that at least $80 \%$ of COPD cases could be avoided by the eradication of cigarette smoking. ${ }^{5}$ Smoking cessation is therefore the first and most important intervention in patients with COPD as it is the only intervention that effectively slows down the accelerated decline in lung function. ${ }^{6}$ Furthermore, smoking cessation reduces symptoms of cough and sputum, improves health status and reduces exacerbations of COPD. ${ }^{7}$ Patients with COPD therefore have a greater and more urgent need to stop smoking than smokers without this disease. ${ }^{8}$ Yet, the proportion of current smokers is higher among people with COPD than in the general population. ${ }^{9}$ In England, for example, more than one-third of patients with COPD still smoke. ${ }^{10}$

The best treatment to help smokers quit smoking according to UK and international clinical guidelines is a combination of pharmacotherapy and behavioural support. ${ }^{8} 11$ Effective pharmacotherapies are bupropion, nicotine replacement therapy (NRT; delivered, eg, through nicotine gum or patch) and varenicline. Varenicline, a selective $\alpha 4 \beta 2$ nicotine acetylcholine receptor partial agonist, is the newest drug on the market; it was introduced in the UK in December 2006 and has been recommended by the National Institute of Health and Care Excellence (NICE) as a treatment option since July $2007 .^{12}$

Varenicline has been shown in experimental studies to be more effective than bupropion and nicotine patches in promoting smoking cessation in the general smoking population. ${ }^{13} 14$ Furthermore, varenicline is the only drug with proven long-term efficacy in smokers with COPD; a recent trial showed a fourfold increase in continuous abstinence over a period of 12 months in users of varenicline compared with placebo. ${ }^{15}$ Other medications have failed to prove efficacy over a period longer than 6 months in this group of smokers. ${ }^{16}$ Varenicline has thus become the most frequently prescribed smoking cessation medication after NRT in England. ${ }^{17}$

The safety profile of varenicline was initially established using standard approaches to pharmacovigilance. However, subsequent postmarketing reports have raised concerns about the safety of varenicline with regard to cardiovascular and neuropsychiatric events. For example, one meta-analysis reported a small, but significantly increased risk of serious adverse cardiovascular events in users of varenicline. ${ }^{18}$ Possible mechanisms for an increased cardiovascular risk may relate to varenicline's action on $\alpha 3 \beta 4$ receptors in the peripheral ganglia and subsequent release of acetylcholine, release of catecholamines and the central influence of $\alpha 4 \beta 2$ and $\alpha 7$ receptors on blood pressure homoeostasis. ${ }^{19}$ A possible mechanism for an increased neuropsychiatric risk may in part be explained by smoking itself, that is, by neuropsychiatric conditions that already existed prior to the quit attempt or other smoking-related conditions that are themselves associated with an increased neuropsychiatric risk. ${ }^{20} 21$ Nevertheless, the European Medicines Agency and the US Food and Drug Administration issued warnings that serious neuropsychiatric symptoms had occurred in smokers trying to stop with varenicline including changes in behaviour, agitation, depressed mood, suicidal ideation, and attempted and completed suicides. ${ }^{22} 23$

Many recent studies conducted outside of clinical trials in the general smoking population did not, however, find any increased risk of cardiovascular and neuropsychiatric events in varenicline users ${ }^{24-31}$ (see table 1 for an overview of previous studies). Further research is needed given the large number of smokers around the globe who wish to stop smoking and who are being prescribed varenicline. There is also a need to assess its safety in important subgroups of the smoking population-in particular smokers with COPD who may be eminently vulnerable for side effects of drugs because they are at increased risk of comorbidity, including cardiovascular and neuropsychiatric diseases. 632

Randomised controlled trials and even meta-analyses are often underpowered to detect rare, serious adverse events. ${ }^{33}$ Large general practice (GP) databases, which routinely collect data on prescribed treatments and disease outcomes are an alternative, promising approach to investigate rare events. An advantage of large GP databases is the higher generalisability of findings compared with randomised controlled trials where patients need to provide informed consent for participation and where selection of patients occurs through exclusion criteria, resulting in a population which is healthier and less vulnerable than the general population. A disadvantage of most GP databases is that analyses are restricted to routinely collected data (and may therefore be incomplete and/or inaccurate). Even more important, analyses of these non-randomised data may be biased because of confounding by indication, that is, the fact that smokers who self-select to use a particular smoking cessation medication differ from patients not using this medication with regard to the factors that have an effect on the outcome. For example, it has been shown that smokers who use pharmacotherapy are heavier and more severely addicted smokers than those who try to quit without pharmacotherapy. ${ }^{34-36}$ To reduce this bias in the current context, adverse events in patients using varenicline need to be compared with patients using other drugs with the indication smoking cessation, such as bupropion or NRT on prescription $(\mathrm{Rx})$. In addition, statistical adjustment for confounders is important. Previous studies successfully used GP databases to assess the safety of smoking cessation medications (see table 1) and the methodology used therein can inform new studies.

The aim of this study is to assess the safety of varenicline using data from the QResearch GP database. Our primary research question is: in smoking patients from general practice, is the use of varenicline for smoking cessation compared with bupropion and NRT Rx 
Table 1 Overview of previous studies on cardiovascular and neuropsychiatric events in users of varenicline and bupropion

\section{Author (year}

\section{of publication) Study type}

Svanström $(2012)^{29}$ Retrospective cohort study using national patient registry

\section{Exposure}

Varenicline vs.

bupropion

Meta-analysis of 14

Prochaska $(2012)^{26}$ randomised controlled trials

Singh (2011) $)^{18}$ Meta-analysis of 14 randomised controlled trials

Thomas

$(2013)^{28}$

Retrospective cohort study using GP database

Pasternak $(2013)^{27}$

Retrospective cohort study using national patient registry

Meyer (2013) ${ }^{31}$ Retrospective cohort study using a military health system claims database

Buggy (2013) ${ }^{30}$ Retrospective cohort study in patients who received a prescription of varenicline by their GP

Varenicline

\section{Outcome}

Cardiovascular event (acute coronary syndrome, ischaemic stroke, or cardiovascular death) Acute coronary syndrome

Ischaemic stroke

Cardiovascular death

Varenicline vs. placebo

serious adverse event (myocardial Not reported

infarction, unstable angina, coronary

revascularisation, coronary artery disease,

arrhythmias, transient ischaemic attacks, stroke,

sudden death or cardiovascular-related death, or congestive heart failure)

Varenicline vs. placebo Cardiovascular event (ischemia, arrhythmia

congestive heart failure, sudden death or cardiovascular-related death)

Fatal or non-fatal self-harm

Varenicline vs NRT

Bupropion vs. NRT

Varenicline vs NRT

Bupropion vs NRT

Treated depression

Psychiatric adverse event (emergency department visit or in-patient admission with a psychiatric diagnosis)

ICD-9 coded diagnosis of schizophrenia, non-organic psychoses, suicide attempt, or drug-induced/transient mental-, mood-

delusional-, anxiety-, personality-, post-traumaticor depressive disorders.

GP-reported depression, anxiety, aggression,

(without comparison)
Incidence of

events per 1000 Relative event rates

patients per year

Varenicline: 6.9 ,

bupropion: 7.1

Varenicline: 4.7,

bupropion: 3.9

Varenicline: 1.9 ,

bupropion: 2.5

Varenicline: 0.4 ,

bupropion: 0.7

(95\% Cls)

$\mathrm{HR}=0.96$ (0.67 to 1.39$)$

$\mathrm{HR}=1.20(0.75$ to 1.91$)$

$\mathrm{HR}=0.77(0.40$ to 1.48$)$

$\mathrm{HR}=0.51(0.13$ to 2.02$)$

$\mathrm{RR}=1.40$ (0.82 to 2.39$)$

Varenicline: 10.6, placebo: 8.2

Varenicline: 2.6, bupropion: 2.5

NRT: 3.6

Varenicline: $57.5, \quad \mathrm{HR}=0.75$ (0.65 to 0.87 ) bupropion: 41.6 ,

NRT: 77.5

Not reported $\mathrm{HR}=0.63$ (0.46 to 0.87$)$

$\mathrm{HR}=0.85(0.55$ to 1.30$)$

Varenicline: $18.1, \quad \mathrm{HR}=1.14$ (0.56 to 2.34$)$ NRT: 15.8

NA

NA. The hazard during the observation period was constant for all events except for anxiety

Continued 


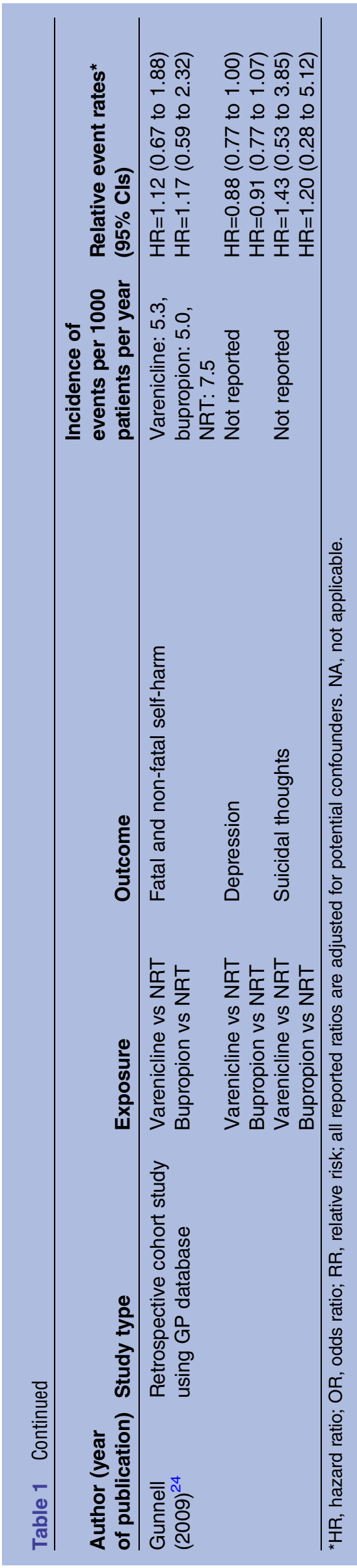

associated with an increased risk of cardiovascular and/ or neuropsychiatric events? This research question will also be addressed in the subgroup of smokers with COPD.

\section{METHODS AND ANALYSIS}

We will conduct a retrospective cohort study involving all adult patients from QResearch who used varenicline, bupropion, or an NRT Rx between 1 January 2007 and 31 December 2012. QResearch is a very large, validated electronic GP database; it includes data from the anonymised health records of over 13 million patients from 753 general practices from across England that use the EMIS software system (http://www.qresearch.org). QResearch has been used for various research studies, including studies of the incidence and risk neuropsychiatric and cardiovascular events ${ }^{37-41}$ (for a complete and up-to-date list of studies visit: http://www.qresearch.org/ SitePages/publications.aspx). External validation studies showed that studies using this database yield similar results as those using other databases such as the Clinical Practice Research Datalink (CPRD) ${ }^{42}{ }^{43}$ and The Health Improvement Network (THIN) database. ${ }^{44}$ Several specific methods described in this protocol are based on the methods used in previous studies on the association between varenicline and neuropsychiatric events which used CPRD, ${ }^{24} 28$ because this database is similar to QResearch (for a detailed comparison see, eg, refs. 42 and 43). Hence, we will use methods which have been established by other researchers in the current context. Furthermore, we will be able to compare some of the results from our study with the previous studies using CPRD.

\section{Inclusion and exclusion criteria}

Only patients meeting all of the following criteria will be included:

- Registered for $>12$ months prior to data extraction at any time during the study period, including those who die or de-register during their study period.

- Prescription of either varenicline alone, bupropion alone, or NRT Rx alone between 1 January 2007 and 30 June 2012. The date of first prescription of one of these drugs will define the individuals entry date to the cohort. We chose this start date because varenicline was introduced to the UK market in December 2006. The end date will be the latest date of data extraction from the database. The latest date of a patient to be included will be 30 June 2012 in order to have 6-month follow-up data until the end of the study period (31 December 2012).

- Aged 18-100 years. We will include only patients over the age of 18 because varenicline and bupropion are only licensed for use in adults in the UK.

Patients with one or more of the following criteria will be excluded: 
- Patients with less than 1 year of QResearch records before their first recorded prescription to ensure that data are of adequate quality.

- Temporary residents.

- Use of one of the three smoking cessation drugs during 12 months prior to the start date of the study (ie, in the period from 1 January 2006 to 31 December 2006) to assure an adequate washout period, so that any adverse events are not attributable to the previous use of these drugs.

- Prescription of a combination of smoking cessation drugs or prescription of another smoking cessation drug during the 6-month follow-up after the patient's entry date to single out adverse events of the three distinct drugs.

The analysis in the subgroup of smokers with COPD will be restricted to patients aged 35 years and over at their individual entry date, with a recorded diagnosis of COPD and recordings of spirometry and Medical Research Council (MRC) dyspnoea score ${ }^{45}$ at any time (all recordings based on appropriated Read codes; see online supplementary appendix). We chose 35 years as the lower age limit for inclusion because COPD is usually not diagnosed before that age. The MRC scale is used as a COPD indicator in the UK National Health Service Quality Outcomes Framework and measures self-perceived disability caused by breathlessness and distinguishes between five levels of severity. ${ }^{45}$ The score will be used in our analyses as an indicator of disease severity.

\section{Exposure measures}

Patients will be categorised into three exposure groups: (1) varenicline alone, (2) bupropion alone or (3) NRT Rx alone (=reference group: any form of NRT, such as nicotine patch, inhaler, nasal spray, gum, sublingual tablet or lozenge), based on the drug they were first prescribed. In the UK, all three drugs are only licensed for use to aid smoking cessation (as described and assessed in a previous study using a different GP database $^{28}$ ). The exposure category will be defined by this initial drug and patients will be considered exposed to the respective drug and no others. Thus, all patients included in the cohort will be unique. The usual course of treatment is 12 weeks of varenicline, 9 weeks for bupropion and 8-12 weeks for NRT Rx. Start of follow-up will begin for each patient on the date of the first prescription of the smoking cessation medication (which is also the patient's entry date to the cohort) and will end after 6-month follow-up or when reaching the specific outcome of interest (ie, a major neuropsychiatric and/or cardiovascular event). Patients who were lost to follow-up because they left the practice or died will be censored on that date. The duration of 6-month follow-up is based on the suggestion that adverse events from drug use may occur after the treatment is finished.

\section{Outcome measures}

We will consider separate major incident neuropsychiatric and cardiovascular outcomes that occur during 6 months of follow-up (based on appropriate Read codes; see online supplementary appendix) for which a potential association with varenicline use has been suggested. ${ }^{182425}$ As a secondary outcome, we will assess the occurrence of these events during 3 months of follow-up. Neuropsychiatric outcomes include: (1) fatal or non-fatal intentional self-harm and (2) depressive disorder. Cardiovascular outcomes include: (1) ischaemic heart disease (including myocardial infarction and angina); (2) cerebral infarction and haemorrhage; (3) heart failure; (4) peripheral vascular disease and (5) cardiac arrhythmia (including cardiac arrest). Recordings of these neuropsychiatric and cardiovascular events prior to the patient's entry date to the cohort will be considered to account for confounding by indication.

\section{Confounding factors}

Confounding by indication is an important potential source of bias in a safety analysis using observational (ie, non-randomised) data. This form of confounding means that patients who are prescribed a certain treatment may differ in prognostic factors from patients who are prescribed a different treatment or who do not receive any treatment. In the current context, for example, patients using medication for smoking cessation (varenicline or bupropion) may differ from patients who try to stop smoking without medication. They may have been more exposed to tobacco and are therefore more at risk of cardiovascular events. In the current study, we will first of all reduce the risk of confounding by indication by comparing users of varenicline or bupropion with users of NRT Rx.

Given that concerns have been raised about varenicline adverse events, it is possible that patients with a history of cardiovascular or neuropsychiatric disease or risk factors may be less likely to be prescribed varenicline. Similarly, bupropion is contra-indicated in certain patient subgroups and there is a caution against prescribing in others. Therefore, an extensive list of patient-level and practice-level characteristics will be considered for inclusion as potential confounders in the analyses. These include the following variables measure at or prior to the patient's entry date to the cohort: age, sex, Townsend index of multiple deprivation, ${ }^{46}$ Strategic Health Authority of the GP practice, relevant comorbidities from the Charlson Index ${ }^{47}$ (ie, COPD, diabetes, peptic ulcer disease, renal disease, rheumatological disease or cancer) and alcohol misuse. For the subgroup analysis in smokers with COPD only, we also added MRC dyspnoea score as a marker for COPD severity. As previously mentioned, any recordings of the neuropsychiatric and cardiovascular diseases that occurred prior to the patient's entry date to the cohort will also be considered. 


\section{Sample size calculation}

We will consider HRs of 1.5 or higher as clinically meaningful, indicating a $50 \%$ or higher increased risk of cardiovascular or neuropsychiatric events in users of varenicline compared with NRT Rx. The sample size calculation is based on detecting such an HR in stroke, as previous research showed this to be the outcome with the lowest incidence rate (see table 1). A query of the QResearch database showed an incidence rate of 2/1000 person-years in the general patient population. For the 6-month follow-up period, we will therefore, assume hazard rates of 0.001 and 0.0015 for the NRT Rx and varenicline groups, respectively. Furthermore, the calculation should take into account a higher prevalence of NRT Rx usage than varenicline. A recent study using the English Clinical Practice Research Datalink (CPRD) reported a ratio of 2.6:1 for NRT Rx versus varenicline. ${ }^{28}$ Therefore, using the method by Freedman ${ }^{48}$ for computing the power of the log-rank test, we find that the required number of NRT Rx and varenicline users should be 128798 and 49 538, respectively, to achieve $80 \%$ power to obtain a significant result at $\alpha=0.05$ (two-tailed).

It should be noted that the sample size for the current study will be given by the actual number of patients from the QResearch database who received a prescription of either varenicline, bupropion or NRT Rx during our study period. Once we have received the data set and know these numbers, we can re-compute the statistical power of all seven events under the above assumptions.

\section{Statistical analyses}

We will compare baseline differences of potential confounding factors between the three exposure groups (varenicline, bupropion and NRT Rx). Kaplan-Meier survival curves will be generated and examined for the three groups. We will then use Cox proportional hazards regression models to assess the association between medication use and each of the above mentioned main outcomes, adjusted for the aforementioned confounding factors. The exposure group factor will first be tested with a likelihood ratio test (based on two degrees of freedom). In addition, we will report HRs with 95\% CIs, with days since start of the treatment as the time scale. HRs will be calculated for varenicline and bupropion with NRT Rx as a reference. The proportional hazards assumption will be assessed by a $\chi^{2}$ test for the interaction between treatment status and the underlying time scale. Start of the follow-up will begin for each patient on the date of the first prescription of the smoking cessation medication and will end after 6-month follow-up or when reaching the specific outcome of interest. Patients will be censored who died during follow-up, who left their practice, or reached the end of the follow-up period.

In an explorative analyses we will assess whether the risk of cardiovascular and neuropsychiatric events differ according to sex, by testing the interaction terms in the Cox models between medication use and sex. In case of significant and clinically relevant differences, results will be reported separately for males and females.

We will use propensity score analysis ${ }^{49}$ as an additional analytic approach to account for potential confounding by indication. In multiple logistic regression models medication use (with varenicline vs NRT Rx as dependent variable in the first model and bupropion vs NRT $\mathrm{Rx}$ in the second model) will be regressed on the aforementioned potential confounders. The resulting predicted probability values for medication use (possible range $0.0-1.0$ ) will be used as propensity score for using one drug versus the other. In order to estimate how much of the variation in medication use can be explained by the potential confounders, we will calculate the area under the receiver operating characteristics curve. The possible value from this analysis will be between 0.5 (indicating no association between the propensity score and medication use) and 1.0 (indicating that medication use can be completely explained by the propensity score). We will then trim the sample by excluding patients with a propensity score corresponding with the 2.5th centile or lower in the varenicline, respectively bupropion group and by excluding patients with a propensity score corresponding with the 97.5 th centile or higher in the NRT Rx group. This trimming is intended to exclude patients from the subsequent analyses which used a form of medication strongly contrary to expectation (eg, a patient who had most of the characteristics associated with the use of varenicline but who used NRT Rx) and may therefore reduce residual confounding. ${ }^{50}$ In the propensity score analyses we will then match patients using varenicline to patients using NRT $R x$ in a fixed 1:1 ratio by using the nearest neighbour algorithm ${ }^{51}$ (MatchIt package in $\mathrm{R}^{52}$ ). Likewise, we will match bupropion users with NRT Rx users. In these two matched samples, we will again use Cox proportional hazards regression models to assess the association between medication use and each of the above mentioned main outcomes. HRs will be calculated for varenicline and bupropion with NRT Rx as a reference.

Another approach we will consider to account for potential confounding by indication is an instrumental variable analysis. ${ }^{53}$ An instrumental variable is assumed to resemble the tossing of a coin to assign patients to a treatment in randomised controlled trials. As such, it provides a method to obtain an unbiased estimate of the association between medication use and adverse events in the current study. A pre-requisite is to find a valid and strong instrumental variable, which is often difficult, because this variable must fulfil the following criteria: (1) it must be a predictor of the treatment (in our case the use of varenicline, bupropion or NRT Rx); (2) it must not be directly related to the outcome (cardiovascular or neuropsychiatric events) except through the effect of the treatment and (3) it must not be associated with measured or unmeasured confounders. We will therefore explore variables that fulfil the above criteria. A potential instrumental variable is the physician's 
prescribing preference, which is often used in prescription drug research with observational data. ${ }^{54}$

All analyses will be repeated for the secondary outcome (ie, the occurrence of neuropsychiatric and cardiovascular events during 3 months of follow-up) and in the subgroup of smokers with COPD.

Data will be analysed with complete cases, that is, patients with missing data on one of the variables age, sex, Townsend index, Strategic Health Authority of the GP practice will be excluded. Only in the subgroup analyses of patients with COPD, those patients with COPD with missing data on spirometry or the MRC dyspnoea score will also be excluded. The percentage missing data for these variables will be reported. Data on recorded diseases that occurred prior to a patient's entry date to the cohort and which will be used as potential confounders will be coded as ' 1 ' if the disease occurred or otherwise ' 0 ': diabetes, peptic ulcer disease, renal disease, rheumatological disease, cancer, alcohol misuse, and the neuropsychiatric and cardiovascular diseases of outcome.

All analyses will be undertaken in R (V.3.0.2 or later). Codes used in $\mathrm{R}$ will be reported as supplementary material of publications. All statistical tests will be twosided with $\mathrm{p}<0.05$ indicating significance.

\section{ETHICS AND DISSEMINATION}

This protocol has been independently peer-reviewed by the QResearch Scientific Board and meets the requirements of the Trent research ethics committee. Two articles are planned to be submitted to international peerreviewed journals: (1) results from the general smoking population and (2) results from the subgroup analysis in smokers with COPD. The reporting will be in accordance with the Strengthening the Reporting of Observational Studies in Epidemiology (STROBE) criteria. ${ }^{55}$ The R-code from the data analyses will be published as supplementary material. The findings from these articles are planned to be presented at relevant national and international healthcare/academic conferences.

\section{Author affiliations \\ ${ }^{1}$ Department of Family Medicine, CAPHRI School for Public Health and Primary Care, Maastricht University Medical Centre, Maastricht, The Netherlands \\ ${ }^{2}$ Cancer Research UK Health Behaviour Research Centre, University College London, London, UK \\ ${ }^{3}$ Allergy and Respiratory Research Group, Centre for Population Health Sciences, The University of Edinburgh, Edinburgh, UK \\ ${ }^{4}$ MHeNS School for Mental Health and Neuroscience, Maastricht University, Maastricht, The Netherlands \\ ${ }^{5}$ Division of General Internal Medicine and Primary Care, Brigham and Women's Hospital/Harvard Medical School, Boston, Massachusetts, USA}

Acknowledgements All authors would like to thank Prof Julia Hippisley-Cox for her advice and support in conducting this study.

Contributors DK had the original idea for this study, drafted its funding application and this article. All other authors contributed to the conception and design of the study, revised the article and gave final approval of the version to be published.
Competing interests DK received an unrestricted grant from Pfizer for a smoking cessation trial. RW received grants, personal fees and non-financia support from Pfizer, GSK and J\&J, and personal fees from Novartis. OCPVS received an unrestricted research grant from Pfizer. AS is supported by The Commonwealth Fund, a private independent foundation based in New York City.

Funding This work was supported by a QInnovation Award (provided by the software provider EMIS and the University of Nottingham)

Provenance and peer review Not commissioned; externally peer reviewed.

Data sharing statement The R-code from the data analyses will be published as online supplementary material.

Open Access This is an Open Access article distributed in accordance with the Creative Commons Attribution Non Commercial (CC BY-NC 4.0) license, which permits others to distribute, remix, adapt, build upon this work noncommercially, and license their derivative works on different terms, provided the original work is properly cited and the use is non-commercial. See: http:// creativecommons.org/licenses/by-nc/4.0/

\section{REFERENCES}

1. World Health Organisation (WHO). WHO report on the global tobacco epidemic. Warning about the dangers of tobacco. Geneva, 2011.

2. Jha $P$, Ramasundarahettige $C$, Landsman $V$, et al. 21st-century hazards of smoking and benefits of cessation in the United States. N Engl J Med 2013;368:341-50.

3. Thun MJ, Carter BD, Feskanich D, et al. 50-year trends in smoking-related mortality in the United States. $N$ Engl J Med 2013;368:351-64.

4. World Health Organisation (WHO). Global burden of disease. http:// www.who.int/mediacentre/factsheets/fs310/en/index.html (accessed 24 Apr 2012). Secondary Global burden of disease. http://www.who. int/mediacentre/factsheets/fs310/en/index.html (accessed 24 Apr 2012).

5. Eisner MD, Anthonisen N, Coultas D, et al. An official American Thoracic Society Public Policy Statement: novel risk factors and the global burden of chronic obstructive pulmonary disease. Am J Respir Crit Care Med 2010;182:693-718.

6. Global Initiative for Chronic Obstructive Lung Disease. Global strategy for the diagnosis, management, and prevention of chronic pulmonary disease. Revised 2011. http://www.goldcopd.com (accessed 24 Apr 2012). Secondary Global strategy for the diagnosis, management, and prevention of chronic pulmonary disease. Revised 2011. http://www.goldcopd.com (accessed 24 Apr 2012).

7. Pride NB. Smoking cessation: effects on symptoms, spirometry and future trends in COPD. Thorax 2001;56(Suppl 2):ii7-10.

8. Tønnesen P, Carrozzi L, Fagerstrom KO, et al. Smoking cessation in patients with respiratory diseases: a high priority, integral component of therapy. ERS task force guideline. Eur Respir J 2007;29:390-417.

9. Shahab L, Jarvis MJ, Britton J, et al. Prevalence, diagnosis and relation to tobacco dependence of chronic obstructive pulmonary disease in a nationally representative population sample. Thorax 2006;61:1043-7.

10. Simpson CR, Hippisley-Cox J, Sheikh A. Trends in the epidemiology of chronic obstructive pulmonary disease in England: a national study of 51804 patients. Br J Gen Pract 2010;60:e277-84.

11. National Institute for Health and Clinical Excellence (NICE) Smoking cessation services in primary care, pharmacies, local authorities and workplaces, particularly for manual working groups, pregnant women and hard to reach communities. London, 2008.

12. National Institute for Health and Clinical Excellence. NICE technology appraisal guidance 123. Varenicline for smoking cessation. London, 2007.

13. Cahill K, Stead Lindsay F, Lancaster T. Nicotine receptor partial agonists for smoking cessation. Cochrane Database Syst Rev 2012; (4):CD006103.

14. Aubin $\mathrm{HJ}$, Bobak A, Britton JR, et al. Varenicline versus transdermal nicotine patch for smoking cessation: results from a randomised open-label trial. Thorax 2008;63:717-24.

15. Tashkin DP, Rennard S, Hays JT, et al. Effects of varenicline on smoking cessation in patients with mild to moderate COPD. Chest 2011;139:591-9.

16. Warnier MJ, Riet EE, Rutten FH, et al. Smoking cessation strategies in patients with chronic obstructive pulmonary disease. Eur Respir $J$ 2013;41:727-34. 
17. Kotz D, Fidler JA, West R. Did the introduction of varenicline in England substitute for or add to the use of other smoking cessation medications? Nicotine Tob Res 2011;13:793-9.

18. Singh S, Loke YK, Spangler JG, et al. Risk of serious adverse cardiovascular events associated with varenicline: a systematic review and meta-analysis. CMAJ 2011;183:1359-66.

19. Harrison-Woolrych M, Maggo S, Tan M, et al. Cardiovascular Events in Patients taking Varenicline. Drug Saf 2012;35:33-43.

20. Farrell $\mathrm{M}$, Howes S, Bebbington $\mathrm{P}$, et al. Nicotine, alcohol and drug dependence and psychiatric comorbidity: Results of a national household survey. Br J Psychiatry 2001;179:432-7.

21. Hughes JR. Smoking and suicide: a brief overview. Drug Alcohol Depend 2008;98:169-78.

22. European Medicines Agency. European Medicines Agency concludes new advice to doctors and patients for Champix needed. EMEA press office, 14 December 2007

23. US Food and Drug Administration. Varenicline (marketed as Chantix) Information. FDA ALERT [2/1/2008]. US Food and Drug Administration, 2 January 2008.

24. Gunnell D, Irvine D, Wise $L$, et al. Varenicline and suicidal behaviour: a cohort study based on data from the General Practice Research Database. BMJ 2009;339:b3805.

25. Svanström H, Pasternak B, Hviid A. Use of varenicline for smoking cessation and risk of serious cardiovascular events: nationwide cohort study. BMJ 2012;345:e7176.

26. Prochaska JJ, Hilton JF. Risk of cardiovascular serious adverse events associated with varenicline use for tobacco cessation: systematic review and meta-analysis. BMJ 2012;344:e2856.

27. Pasternak B, Svanström H, Hviid A. Use of varenicline versus bupropion and risk of psychiatric adverse events. Addiction 2013;108:1336-43.

28. Thomas $\mathrm{KH}$, Martin RM, Davies NM, et al. Smoking cessation treatment and risk of depression, suicide, and self harm in the Clinical Practice Research Datalink: prospective cohort study. BMJ 2013;347:f5704.

29. Svanström H, Pasternak B, Hviid A. Use of varenicline for smoking cessation and risk of serious cardiovascular events: nationwide cohort study. BMJ 2012;345:e7176.

30. Buggy $\mathrm{Y}$, Cornelius V, Fogg C, et al. Neuropsychiatric events with varenicline: a modified prescription-event monitoring study in general practice in England. Drug Saf 2013;36:521-31.

31. Meyer TE, Taylor LG, Xie S, et al. Neuropsychiatric events in varenicline and nicotine replacement patch users in the Military Health System. Addiction 2013;108:203-10.

32. Soriano JB, Visick GT, Muellerova $\mathrm{H}$, et al. Patterns of comorbidities in newly diagnosed COPD and asthma in primary care. Chest 2005;128:2099-107.

33. Sutton AJ, Cooper NJ, Lambert PC, et al. Meta-analysis of rare and adverse event data. Expert Rev Pharmacoecon Outcomes Res 2002;2:367-79.

34. Borland R, Partos TR, Cummings KM. Systematic biases in cross-sectional community studies may underestimate the effectiveness of stop-smoking medications. Nicotine Tob Res 2012;14:1483-7.

35. Shiffman S, Di Marino ME, Sweeney CT. Characteristics of selectors of nicotine replacement therapy. Tob Control 2005;14:346-55.

36. West R, Zhou X. Is nicotine replacement therapy for smoking cessation effective in the "real world"? Findings from a prospective multinational cohort study. Thorax 2007;62:998-1002.

37. Hippisley-Cox J, Fielding K, Pringle M. Depression as a risk factor for ischaemic heart disease in men: population based case-contro study. BMJ 1998;316:1714-19.
38. Hippisley-Cox J, Coupland C, Vinogradova Y, et al. Derivation and validation of QRISK, a new cardiovascular disease risk score for the United Kingdom: prospective open cohort study. BMJ 2007;335:136.

39. Hippisley-Cox J, Coupland C. Effect of combinations of drugs on all cause mortality in patients with ischaemic heart disease: nested case-control analysis. BMJ 2005;330:1059-63.

40. Hippisley-Cox J, Coupland C. Risk of myocardial infarction in patients taking cyclo-oxygenase-2 inhibitors or conventional non-steroidal anti-inflammatory drugs: population based nested case-control analysis. BMJ 2005;330:1366.

41. Hippisley-Cox J, Coupland C, Brindle P. Derivation and validation of QStroke score for predicting risk of ischaemic stroke in primary care and comparison with other risk scores: a prospective open cohort study. BMJ 2013;346:f2573.

42. Reeves D, Springate DA, Ashcroft DM, et al. Can analyses of electronic patient records be independently and externally validated? The effect of statins on the mortality of patients with ischaemic heart disease: a cohort study with nested case-control analysis. BMJ Open 2014;4:e004952.

43. Vinogradova Y, Coupland C, Hippisley-Cox J. Exposure to bisphosphonates and risk of gastrointestinal cancers: series of nested case-control studies with QResearch and CPRD data. BM 2013;346:f114.

44. Collins GS, Altman DG. An independent and external validation of QRISK2 cardiovascular disease risk score: a prospective open cohort study. BMJ 2010:c2442.

45. Bestall JC, Paul EA, Garrod R, et al. Usefulness of the Medical Research Council (MRC) dyspnoea scale as a measure of disability in patients with chronic obstructive pulmonary disease. Thorax 1999:54:581-6.

46. Townsend $\mathrm{P}$, Phillimore $\mathrm{P}$, Beattie A. Health and deprivation: inequality and the North. London: Croom Helm, 1988.

47. Khan N, Perera R, Harper S, et al. Adaptation and validation of the Charlson Index for Read/OXMIS coded databases. BMC Fam Pract 2010;11:1.

48. Freedman LS. Tables of the number of patients required in clinical trials using the logrank test. Stat med 1982;1:121-9.

49. Stürmer T, Joshi M, Glynn RJ, et al. A review of the application of propensity score methods yielded increasing use, advantages in specific settings, but not substantially different estimates compared with conventional multivariable methods. J Clin Epidemio 2006;59:437-47.

50. Sturmer T, Rothman KJ, Avorn J, et al. Treatment effects in the presence of unmeasured confounding: dealing with observations in the tails of the propensity score distribution--a simulation study. $A m \mathrm{~J}$ Epidemiol 2010;172:843-54.

51. Austin PC. Some methods of propensity-score matching had superior performance to others: results of an empirical investigation and Monte Carlo simulations. Biom J 2009;51:171-84.

52. Ho D, Imai K, King G, et al. Matchlt: Nonparametric preprocessing for parametric causal inference. J Stat Software 2011;42:1-28.

53. Rassen JA, Brookhart MA, Glynn RJ, et al. Instrumental variables I: instrumental variables exploit natural variation in nonexperimental data to estimate causal relationships. J Clin Epidemiol 2009;62:1226-32.

54. Chen Y, Briesacher BA. Use of instrumental variable in prescription drug research with observational data: a systematic review. J Clin Epidemiol 2011;64:687-700.

55. von Elm E, Altman DG, Egger M, et al. The strengthening the reporting of observational studies in epidemiology (STROBE) statement: guidelines for reporting observational studies. J Clin Epidemiol 2008;61:344-9. 